\title{
Exploring Approximations for Floating-Point Arithmetic using UppSAT
}

\author{
Aleksandar Zeljić ${ }^{1}$ \\ Peter Backeman $^{1}$ \\ Christoph M. Wintersteiger ${ }^{2}$ \\ Philipp Rümmer ${ }^{1}$ \\ ${ }^{1}$ Uppsala University \\ ${ }^{2}$ Microsoft Research
}

\begin{abstract}
We consider the problem of solving floating-point constraints obtained from software verification. We present UppSAT — an new implementation of a systematic approximation refinement framework [24] as an abstract SMT solver. Provided with an approximation and a decision procedure (implemented in an off-the-shelf SMT solver), UppSAT yields an approximating SMT solver. Additionally, UppSAT yieldsincludes a library of predefined approximation components which can be combined and extended to define new encodings, orderings and solving strategies. We propose that UppSAT can be used as a sandbox for easy and flexible exploration of new approximations. To substantiate this, we explore several approximations of floating-point arithmetic. Approximations can be viewed as a composition of an encoding into a target theory, a precision ordering, and a number of strategies for model reconstruction and precision (or approximation) refinement. We present encodings of floating-point arithmetic into reduced precision floating-point arithmetic, real-arithmetic, and fixed-point arithmetic (encoded into the theory of bit-vectors in practice). In an experimental evaluation we compare the advantages and disadvantages of approximating solvers obtained by combining various encodings and decision procedures (based on existing, state-of-the-art SMT solvers for floating-point, real, and bit-vector arithmetic).
\end{abstract}

\section{Introduction}

The construction of satisfying assignments of a formula, or showing that no such assignments exist, is one of the most central tasks in automated reasoning. Although this problem has been addressed extensively in research fields including constraint programming, and more recently in Satisfiability Modulo Theories (SMT), there are still constraint languages and background theories where effective model construction is challenging. Such theories are, in partic- 
ular, arithmetic domains such as bit-vectors, nonlinear real arithmetic (or realclosed fields), and floating-point arithmetic; even when decidable, the high computational complexity of such problems turns model construction into a bottleneck in applications such as model checking, test-case generation, or hybrid systems analysis.

In several recent papers, the notion of approximation has been proposed as a means to speed up the construction of (precise) satisfying assignments. Generally speaking, approximation-based solvers follow a two-tier strategy to find a satisfying assignment of a formula $\phi$. First, a simplified or approximated version $\hat{\phi}$ of $\phi$ is solved, resulting in an approximate solution $\hat{m}$ that (hopefully) lies close to a precise solution. Second, a reconstruction procedure is applied to check whether $\hat{m}$ can be turned into a precise solution $m$ of the original formula $\phi$. If no precise solution $m$ close to $\hat{m}$ can be found, refinement can be used to successively obtain better, more precise, approximations.

This high-level approach opens up a large number of design choices, some of which have been discussed in the literature. The approximations considered have different properties; for instance, they might be over- or under-approximations (in which case they are commonly called abstractions), or be nonconservative and exhibit neither of those properties. The approximated formula $\hat{\phi}$ can be formulated in the same logic as $\phi$, or in some proxy theory that enables more efficient reasoning. The reconstruction of $m$ from $\hat{m}$ can follow various strategies, including simple re-evaluation, precise constraint solving on partially evaluated formulas, or randomised optimisation. Refinement can be performed with the help of of approximate assignments $\hat{m}$, using proofs or unsatisfiable cores, or be skipped altogether.

In this paper, we aim at a uniform description and exploration of the complete design space. We focus on the case of (quantifier-free) floating-point arithmetic (FPA) constraints, a particularly challenging domain that has been studied extensively in the SMT context over the past few years [3, 12, 23, $22,13,24]$. To enable uniform exploration of approximation, reconstruction, and refinement methods, as well as simple prototyping and comparative studies, we present UppSAT as a a general framework for building approximating solvers. UppSAT is implemented in Scala, open-source under GPL licence, and allows the implementation of approximation schemes in a modular and high-level fashion, such that different components can be easily combined with various back-ends.

With the help of the UppSAT framework, we explore several ways of approximating SMT reasoning for floating-point arithmetic. The contributions of the paper are:

- a high-level approach to design, implement, and evaluate approximations, presented using the case of floating-point arithmetic constraints; 
- a conceptual and experimental comparison of three different forms of FPA approximation, based on the notions of reduced-precision floatingpoint arithmetic, fixed-point arithmetic, and real arithmetic;

- a systematic comparison of different back-end solvers for the case of reduced-precision floating-point arithmetic.

\subsection{Introductory Example}

In this paper we will use the following formula as running example to illustrate the effects of using different approximations:

Example 1. Consider a floating-point formula $\phi$ over two variables $x$ and $y$ :

$$
y=x+1.75 \wedge y \geq 0 \wedge(x=2.0 \vee x=-4.0)
$$

Note that the formula can be satisfied by the model $m=\{x \mapsto 2.0, y \mapsto 3.75\}$ in both single-precision and double-precision FPA (and a couple of further formats).

We will use the formula to highlight different aspects of the approximations discussed in this paper, in particular approximations using reduced-precision FPA and fixed-point arithmetic.

\subsubsection{Reduced-Precision Floating-Point Arithmetic}

The first form of approximation uses floating-point operations of reduced precision, i.e., with a reduced number of bits for the significant and exponent. Approximations of this kind have previously been studied in [23, 24], and found to be an effective way to boost the performance of bit-blasting-based SMT solvers, since the size of FPA circuits tends to grow quickly with the bit-width. The change of the actual formula lies in decreasing the number of bits used for each variable and operator.

Example 2. We assume reduction to the floating-point $(3,3)$ format, i.e., the format in which 3 bits are used for the significant, and 3 bits for the exponent. The approximate formula $\hat{\phi}_{3,3}$ is obtained by replacing the variables $x$ and $y$ with re-typed variants $x_{3,3}, y_{3,3}$, casting all floating-point literals to the new format, and replacing the addition operator + and comparison predicate $\leq$ with the operator $+_{3,3}$ and the predicate $\geq_{3,3}$ for reduced-precision arguments:

$$
y_{3,3}=x_{3,3}+_{3,3} 1.75_{3,3} \wedge y_{3,3} \geq 0_{3,3} \wedge\left(x_{3,3}=2.0_{3,3} \vee x_{3,3}=-4.0_{3,3}\right)
$$

Even though $\hat{\phi}_{3,3}$ is satisfiable the models are not guaranteed models for the original formula, but only satisfies the reduced precision formula because of over/under-flows and rounding errors when working with only three precision and three significand bits. For example, $\hat{m}=\{x \mapsto 2.0, y \mapsto 4.0\}$ ), satisfies $\hat{\phi}_{3,3}$ because $2.0_{3,3}+3,31.75_{3,3}=4_{3,3}$. 
This could means that the current reduced precision does not allow for representation of the solutions that exists for the full precision formula. Therefore we need to refine the precision, and a simple strategy is to increase the precision of every node by the same amount, yielding:

$$
y_{5,5}=x_{5,5}+_{5,5} 1.75_{5,5} \wedge y_{5,5} \geq 0_{5,5} \wedge\left(x_{5,5}=2.0_{5,5} \vee x_{5,5}=-4.0_{5,5}\right)
$$

which will have model $\{x \mapsto 2.0, y \mapsto 3.75\}$ which is also a model for the original problem.

\subsubsection{Fixed-Point Arithmetic}

As a second relevant case, we consider the use of fixed-point arithmetic as an approximation of FPA. This is done by choosing a fixed number of integral bits and a fixed number of fractional bits, defining the applied fixed-point format, and then recasting each floating-point constraint as a bit-vector constraint: each floating-point operation is replaced with a set of bit-vector operations implementing the corresponding computation over fixed-point numbers.

Example 3. For our example, we can initially choose a representation with 5 integral and 5 fractional bits, i.e., in the $(5,5)$ fixed-point format. We can note that fixed-point $(5,5)$-addition is exactly implemented by bit-vector addition $\oplus_{10}$ over 10 bits, and fixed-point comparison $\geq$ by signed bit-vector comparison $\geq_{s}$ over 10 bits, so that the translation becomes relatively straightforward, resulting in the formula $\hat{\phi}_{5,5}^{F}$ :

$$
\begin{gathered}
y_{10}=x_{10} \oplus_{10} 0000111000_{2} \wedge y_{10} \geq_{s} 0000000000_{2} \wedge \\
\left(x_{10}=0001000000_{2} \vee x_{10}=1110000000_{2}\right)
\end{gathered}
$$

Constants are interpreted as 2's complement numbers with 5 fractional and 5 integral bits, e.g., $1110000000_{2}$ represents the binary number -100.00000 , which is -4.0 in decimal notation.

It can easily be seen that the constraint $\hat{\phi}_{5,5}^{F}$ is satisfied by the model $\hat{m}=$ $\left\{x_{10} \mapsto 0001000000_{2}, y_{10} \mapsto 0001111000_{2}\right\}$, which corresponds to the fixed-point solution $x=2.0$ and $y=3.75$, and to the floating-point solution given in Example 1.

\section{UppSAT - An Abstract Approximating SMT Solver}

UppSAT is an implementation of the systematic approximation refinement framework [24] as an abstract SMT solver. It takes an approximation and a back-end SMT solver to yield an approximating SMT solver. UppSAT can implement a broad range of approximations in a simple and modular way, and it easily integrates off-the-shelf SMT solvers.

The theoretical framework [24] is defined in terms of a monolithic auxiliary theory to solve the original problem. Instead of solving the problem $\phi$ of 


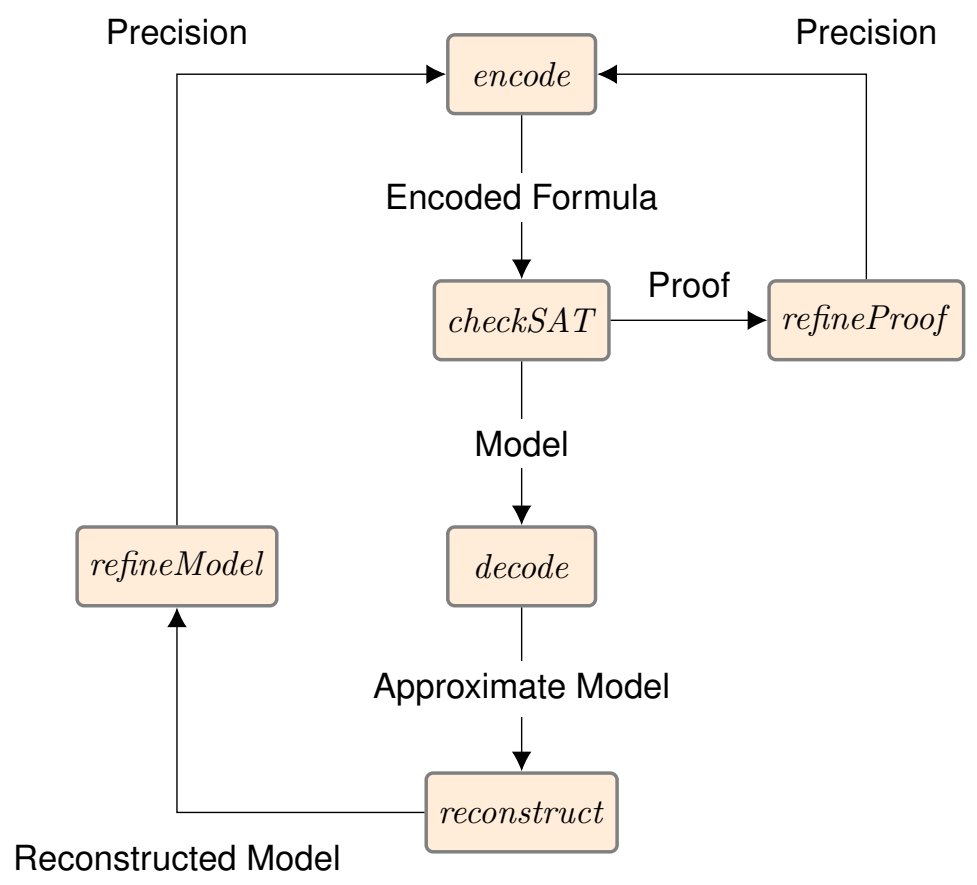

Figure 1. The main components needed to implement approximations in UppSAT, and the flow of data in between them.

theory $T$ directly, the formula $\phi$ is lifted to the formula $\hat{\phi}$ of the approximation theory $\hat{T}$. The formula $\hat{\phi}$ is solved using a decision procedure for $\hat{T}$. $\hat{T}$ enables approximation of the original problem and controlling the degree of approximation. The search for the model is guided by a search through the space of approximations expressible in $\hat{T}$. Lifting the formula $\phi$ to $\hat{T}$ introduces precision as the means of characterizing the degree of approximation. For different values of precision, different approximations of the original formula are obtained. The overall goal is to find a model of an approximation $\hat{m}$ that can be translated back to a model $m$ of the original formula $\phi$.

The solving process can be seen as a two-tier search, in which the search for a sufficiently precise approximation guides the actual model search. Search for the approximation tries to capture essential properties of the model and is performed by the abstract solver. The low-level search is entirely unaware of the high-level aspects. It is performed by the back-end solver which seeks the approximate model. The two tiers of search guide each other in turns, until a solution is found or the search space of approximations has been exhausted. In practice there is no need to implement a solver for the monolithic approx- 
imation theory $\hat{T}$. Instead, UppSAT uses an off-the-shelf SMT solver as the back-end procedure to solve lifted formulas.

The overall goal for the approximation is to produce constraints that are 'easier' to solve than the input constraints. One example, that we consider later, is approximating the theory of FPA using the theory of reals, which is considered 'simpler' because it ignores the rounding behavior and special values of FPA semantics.

A bird's eye view on UppSAT. This paper focuses on the theory of FPA and presents several approximations suitable for solving FPA formulas. We first discuss the general structure of the approximations from the perspective of UppSAT.

An approximation context contains the following components: 1 . an input theory $T$, the language of the problem to solve; 2 . an output theory $\hat{T}$, the language of in which we solve lifted formulas; 3 . a precision domain, the parameters used to indicate degree of approximation; and 4. a precision ordering, defining an order among different approximations.

Given an approximation and a back-end solver, UppSAT takes a set of constraints of the input theory $T$ and produces constraints of the output theory for the back-end solver. Precision regulates the encoding from the input to the output theory, and its domain and ordering are of consequence for encoding, approximation refinement and termination.

The approximation context only determines the setting for the approximation, but does not give the complete picture. For example, fixing an input and an output theory does not uniquely determine the encoding, also the choice of precision domain and the precision ordering are essential for the expressiveness of the approximation. Given an approximation context, to fully define an approximation we also need to define the following components: 1. encoding of the formula based on precision; 2. decoding the values of the approximate model; 3. model reconstruction strategy; 4. model-based refinement strategy; and 5. proof-based refinement strategy. The flow of data between these components can be seen in Fig 1.

Encoding of the formula and decoding of the approximate model are the core of the approximation. These operations describe the two directions of moving between the input and the output theory. The encoding aims to retain the essential properties of the problem while making it easier to solve. The goal of decoding is to translate a model for the approximate constraints to an assignment of the input theory. These two operations are of course closely related (and implemented by the Codec trait).

The purpose of model reconstruction strategy is to transform the decoded model into a model of the original constraints. Sometimes the model of the lifted formula will also be a model for the original formula. However, often this is not the case, and a reconstruction strategy is used to repair the assign- 
ment in an attempt to find a satisfying assignment. Reconstruction strategies can range from simple re-evaluation of the constraints to using a constraint solver or an optimization procedure.

The goal of the model-based and proof-based refinement strategies is to select the approximation for the next iteration based on the available information. For example, given an approximate model and a failed model we can infer which parts of the formula to refine. This is expressed as a new precision value, specifically a precision value which is greater than the previous one according to the precision ordering.

In order to preserve completeness and termination, for the case of decidable theories, we assume that every precision domain contains a top element $T$, and that precision domains satisfy the ascending chain condition (every ascending chain is finite) [24]. By convention, approximation in top precision $T$ corresponds to solving the original, un-approximated constraint with the help of a fall-back solver.

Implementation of these operations can be separated into two layers, a general layer and a theory-specific layer. For example, significant parts of the encoding and decoding are specific to theories involved in the approximation, while the various strategies are mostly theory-independent, except for a few details. Theory-independent layers are abstracted into templates, that provide hook functions for theory-specific details. UppSAT is designed around the mix-and-match principle, to provide a sandbox for testing different approximations with little implementation effort.

Fig. 2 shows the traits (i.e., interfaces) that have to be implemented by approximations in UppSAT. The approximation class takes an object implementing all four traits, and combines them into an approximation to be used by the abstract solver.

Consider an implementation of the reduced precision approximation of FPA in a concise and compact manner. This approximation comprises of dropping FPA-specific elements, such as the rounding modes, and replacing FPA operations by the corresponding real arithmetic operations. in certain cases, a combination of operations may be necessary, e.g., in the case of the fusedmultiply-add operation. In the case of the FPA theory, the approximation could hard-code one rounding mode for all operations, change the variables and operations to have reduced precision, or just omit some of the constraints.

\section{Specifying Approximations in UppSAT}

In this section we show how to specify approximations in UppSAT, ${ }^{1}$ using the example of reduced-precision FPA [24] from Section 1.1.1. It should be remarked that one of the design goals of UppSAT is the ability to define approximations in a convenient, high-level way; the code we show in this section

${ }^{1}$ https://github.com/uuverifiers/uppsat 


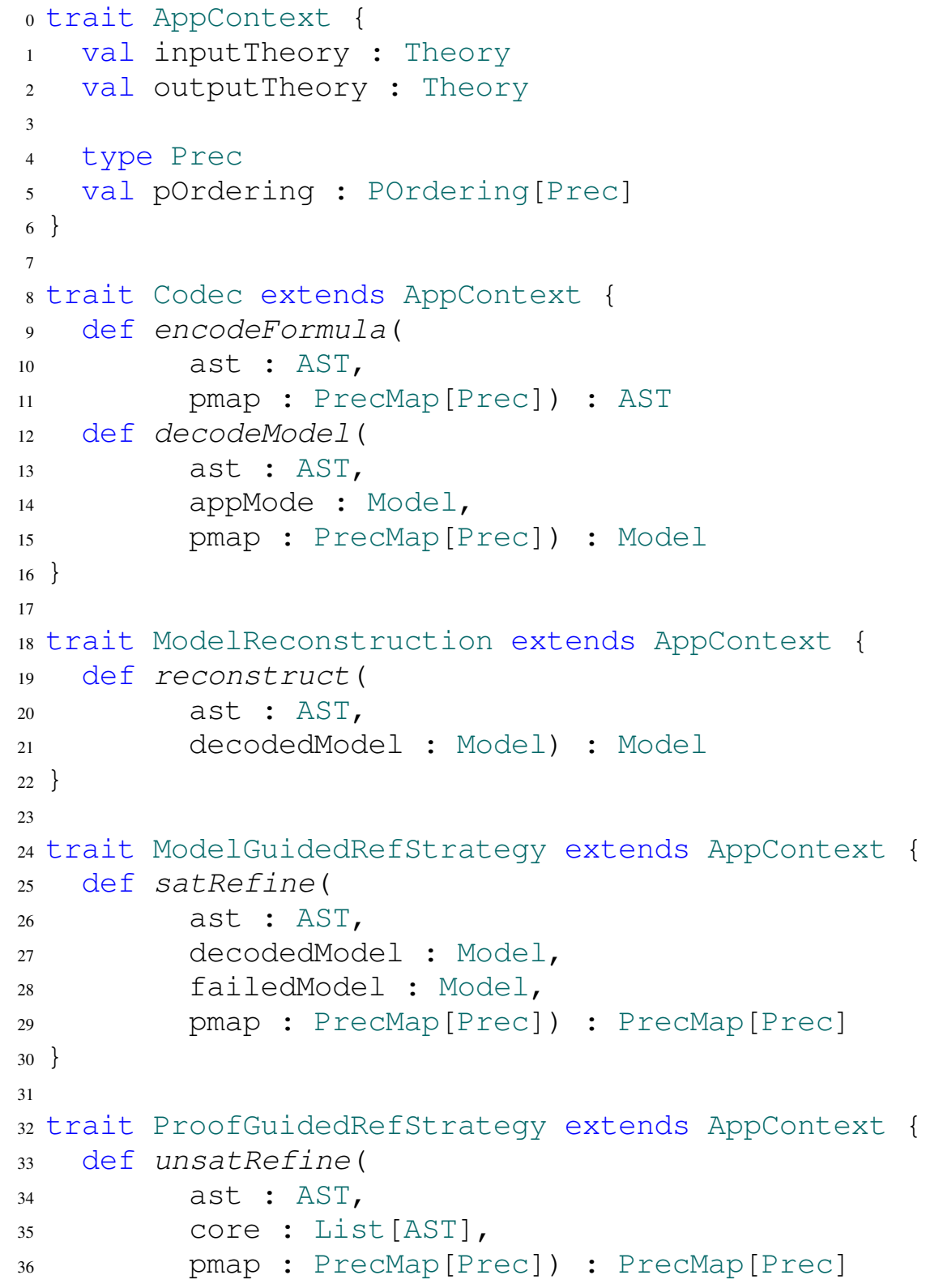

Figure 2. The basic traits neccessary to specify an approximation in UppSAT 


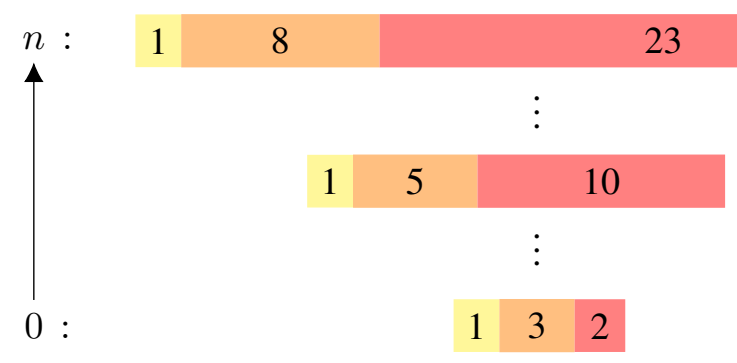

Figure 3. Example of scaling a single precision floating-point sort

is mostly identical to the actual implementation in UppSAT, modulo a small number of simplifications for the purpose of presentation. We will first give an intuition for this particular approximation, before breaking it down into the elements that UppSAT requires.

\subsection{Approximation using Reduced-Precision FPA}

Floating-point numbers are a two-parameter data type, denoted $F P_{e, s}$. The parameters $e$ and $s$ are the number of bits used to store the exponent and the significand in memory, respectively. The IEEE-754 standard specifies several distinct combinations of $e$ and $s$, for example, single precision $F P_{8,24}$ and double precision $F P_{11,53}$ floating-point numbers. And indeed, these are the most commonly used data types to represent real-valued data. Solving FPA constraints typically involves encoding them into bit-vector arithmetic and subsequently into propositional logic, via a procedure called flattening or bit-blasting. The size and complexity of the propositional formula depends on the size of floating-point numbers in memory. Such an encoding of FPA constraints can become prohibitively large very quickly. However, many key values, e.g., special values, one, powers of two, can be represented compactly and exist in floating-point representations that contain very few bits. Therefore, reasoning over single- or double-precision floating-point numbers, for models that involve mostly (or only) these values can be wasteful. Instead, we solve a reduced-precision version of the formula, i.e., we work with Reduced Precision Floating Points (RPFP). Reducing the precision does not affect the structure of the formula, and only changes the sorts of floating-point variables, predicates and operations. Bit-blasting reduced-precision constraints results in significantly smaller propositional formulas, that are still expressive enough to find an approximate solution.

\subsection{Reduced-Precision FPA Approximation in UppSAT}

An approximation in UppSAT consists of several parts: an approximation context (the "approximation core"), a codec, a model reconstruction strategy, 


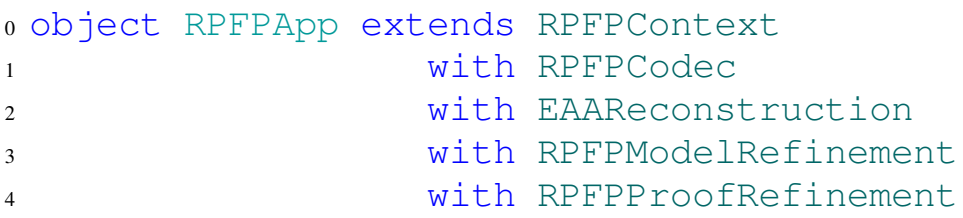

Figure 4. Specification of the reduced precision floating-point approximation as a Scala object.

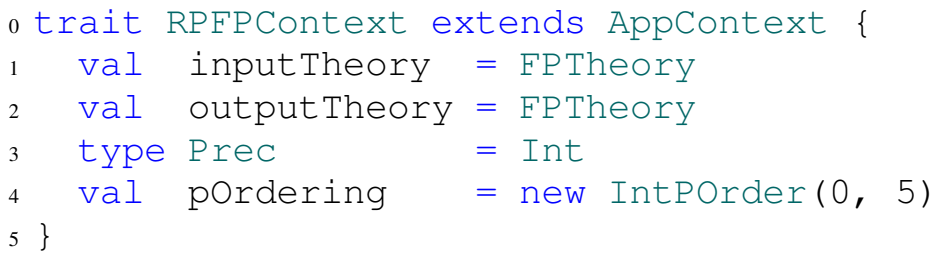

Figure 5. Specifying the approximation context for the reduced precision floatingpoint approximation.

and a refinement strategy for model- and proof-guided refinement. Fig. 4 shows the object RPFPApp implementing the reduced-precision floatingpoint approximation. The approximation object is implemented using Scala mix-in traits (shown in Fig. 2), which enable the modular mix-and-match approximation design. In the following paragraphs, we show the key points of reduced precision floating-point approximation through its component traits.

Approximation context. An approximation context specifies input and output theory, a precision domain and a precision ordering. The reduced-precision floating-point approximation encodes floating-point constraints as scaled-down floating-point constraints. Therefore, both the input and the output theory are the quantifier-free floating-point theory ( EPTheory). The precision uniformly affects both the significand and the exponent, so a scalar data type Prec $=$ Int is sufficient to represent precision. In particular, we choose integers in the range $[0,5]$ as the precision domain with the usual ordering. Fig. 5 shows the specification of RPFPContext the approximation context object for the reduced precision floating-point approximation.

Codec. The essence of approximation takes place in the encoding of the formula, and conversely how the approximate model is decoded. These two operations are implemented by the RPEPCodec trait, shown in Fig. 6 for the case of the reduced-precision FPA. Reduced-precision floating-point approx- 
imation scales-down the sort of floating-point variables and operations, while keeping the high-level structure of the formula. Scaling for operations and variables are performed based on precision values, while predicate nodes are scaled to the largest sort among their children. Constant literals and rounding modes remain unaffected by encoding. The discrepancy in sorts due to individual precisions is removed by inserting $f p$.toFP casts where necessary. The fp.toFP declaration is an SMT-lib function which casts a Floating Point value to a given sort. To ensure internal consistency of the approximate models, all occurences of a variable share the same precision. Predicate scaling requires that the sorts of the arguments are known, i.e., arguments are already encoded when their parent node is encoded. Therefore, we consider a formula as an abstract syntax tree (AST) and use a post-order visit pattern over the formula. UppSAT provides a template trait for such an encoding called PostorderCodec. To implement it, the user needs to define two hook functions: encodeNode and decodeNode.

To encode a node, we scale the sort, pad the arguments, re-instantiate the symbol to the new sort and bundle the new symbol with the padded children. These steps are implemented in the encodeNode hook function, shown in Fig. 6. The details of scaling the sort are shown in the scaleSort auxiliary function. The sort scaling is linear and consists of 6 sorts, starting with the $F P_{3,3}$ up to (and including) the original sort. The cast function adds a floating-point cast fp.toFP between the parent and the child node where necessary. Implementation of the functions cast and encodeSymbol is straightforward and omitted in the interest of brevity.

After the back-end solver returns a model of the approximate constraints, it needs to be decoded. Decoding is essentially casting variable assignments to their sort in the original formula. For example, suppose a formula $\phi$ over variables $x$ and $y$ of sort $F P_{8,24}$ is encoded to the formula $\hat{\phi}_{3,3}$ (as in Ex. 2), yielding a model $\hat{m}=\left\{x \mapsto 0_{3,3} ; y \mapsto 1_{3,3}\right\}$. Decoding will cast these values from the model of approximate constraints and translate them to the same values, but in their full-precison sort, resulting in a variable assignment $m=$ $\left\{x \mapsto 0_{8,24} ; y \mapsto 1_{8,24}\right\}$. Special values are also decoded by re-instantiating them in the original sort. Other values are decoded by adding the missing bits to their representation. The missing bits in the encoded formula are implicitly set to zero. To decode the significant, the missing zero bits are simply reinserted. Padding the exponent requires some attention due to the details of the IEEE-754 standard. The values of the exponent are stored with an added bias value, which is dependent on the exponent bit-width. To pad the exponent, we first remove the bias of the exponent in reduced precision, and then add the bias of full-precison FP. (Subnormal floating-point values require more attention.)

PostOrderCodec implements the decodeModel function through the decodeNode hook function. The hook function is applied to all the values 


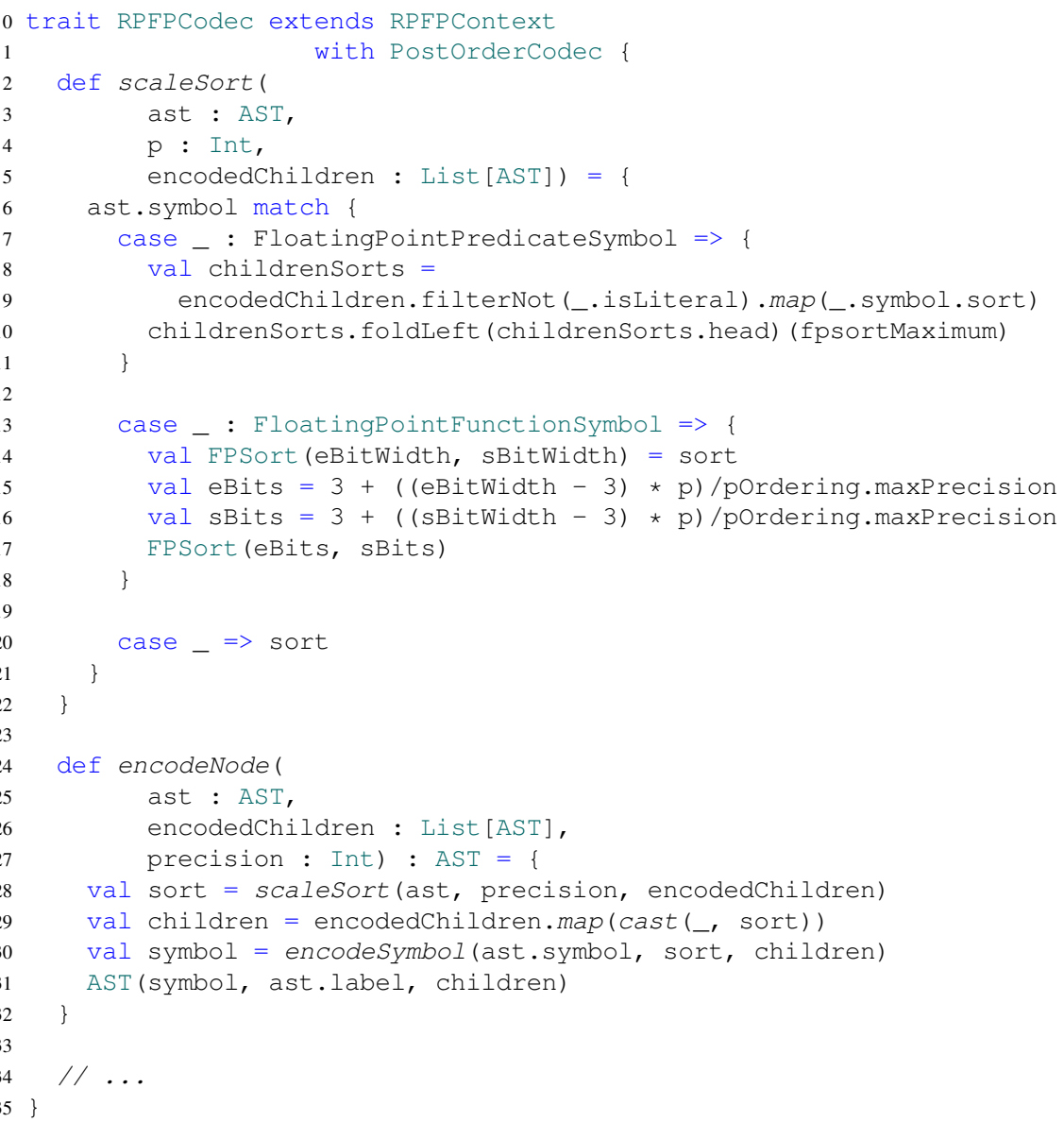

Figure 6. Implementation of the reduced-precision encoding. 


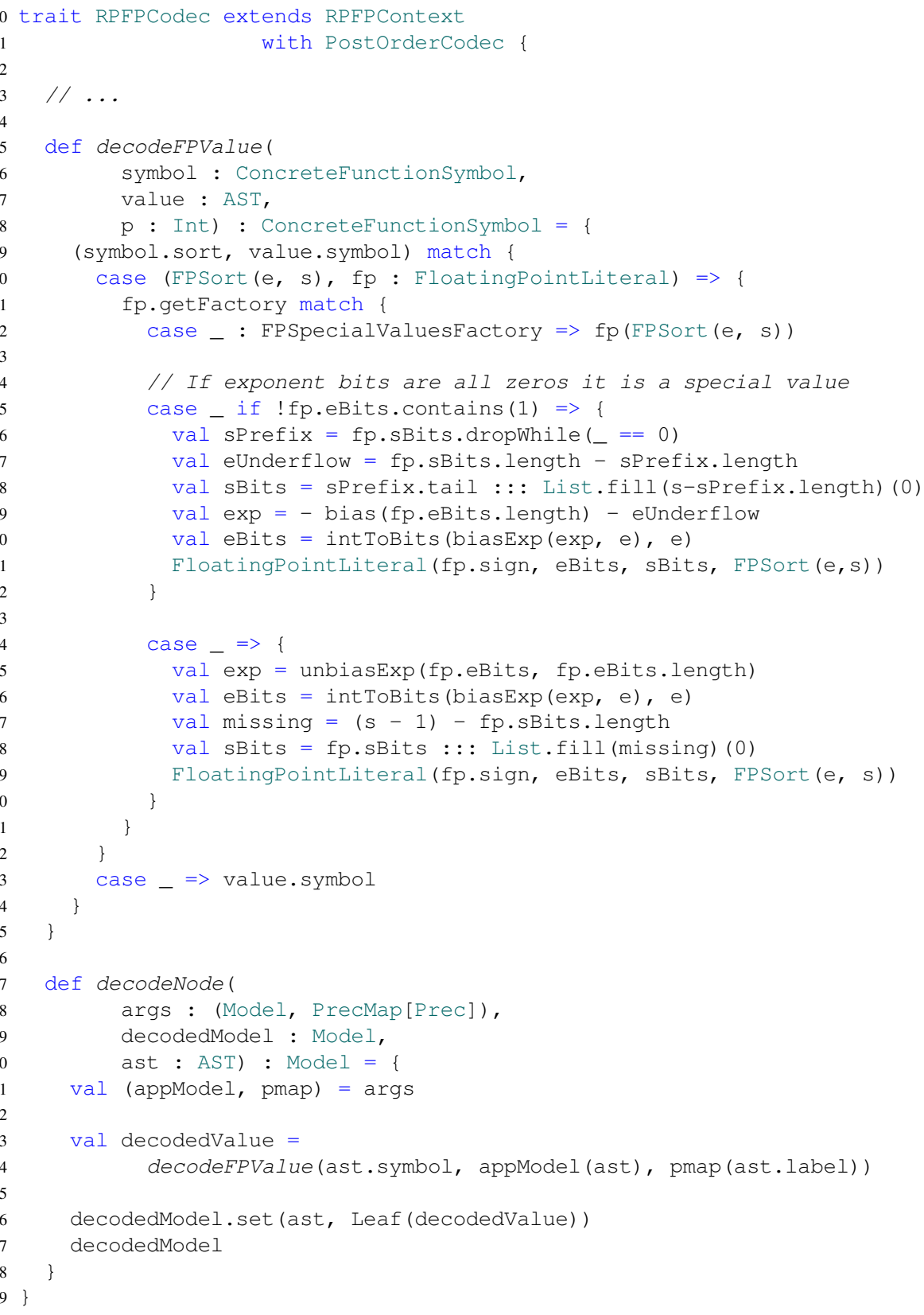

Figure 7. Implementation of the reduced-precision decoding (part of the trait in Fig. 6.) 
in the model of the approximate constraints. The decoding of the values is performed by the decodeFPValue function, all shown in Fig. 6.

Model reconstruction strategy specifies how to obtain a model of the input constraints starting from the decoded model. A simple strategy to obtain a reconstructed model is to satisfy the same Boolean constraints (constraints which are true or false, e.g., equalities, inequalities, predicate) as the approximate model, i.e., to try and satisfy the Boolean structure in the same way. We call those constraints critical atoms. However, due to the difference in semantics, values of the decoded model are not guaranteed to satisfy them. Typically, the rounding error, significantly larger in reduced precision FPA, accumulates and changes the value of critical atoms under the original semantics. Therefore, evaluation of critical atoms under the original semantics is necessary to ensure that the model satisfies the original formula. In fact, rather than evaluating the critical atoms simply as a verification step, evaluation can be used to infer the error-free values under the original semantics. Starting from an empty candidate partial model, the constraints are evaluated in a bottom-up fashion. Thus, the reconstruction can be defined by defining the reconstruction of a single node in the reconstruct Node hook function, shown in Fig. 8.

The key to a good reconstruction strategy is propagation. Certain constraints allow more information to be propagated than others. For example, equality $x=y+z$ uniquely determines the value of $x$ if the values of $y$ and $z$ are known and the equality is known to hold. Whereas, an inequality for example, allows for less propagation. The decoded model contains the information which critical atoms need to be satisfied. The critical atoms combined with a bottom-up evaluation, allow propagation to take place, by applying equalityas-assignment; if the following conditions are satisfied: 1 . the equality is true in the approximate model, 2 . its left- or right-hand side is a variable that is currently unassigned in the candidate model, and 3 . the value of the other side is defined in the candidate model, then the variable can be assigned the value of the other side in the candidate model. Equality-as-assignment is crucial for elimination of rounding errors due to the RPFP encoding. Note that this reconstruction strategy can fail if cyclic dependencies exist among the constraints.

An important aspect of the reconstruction strategy is the order of evaluation. Bottom-up evaluation, bar equality-as-assignment, requires that all the sub-expressions have a value in the candidate model. The base case are variables which might be undefined in the candidate model. If they are undefined in the candidate model when they are needed for evaluation, they are assigned the value from the decoded model. This means, that evaluation of inqualities ahead of equalities might prevent equality-as-assignment to take place. Therefore, we wish to evaluate predicates in an order such that equality-asassignment enabled critical atoms are evaluated first. Therefore we separate 


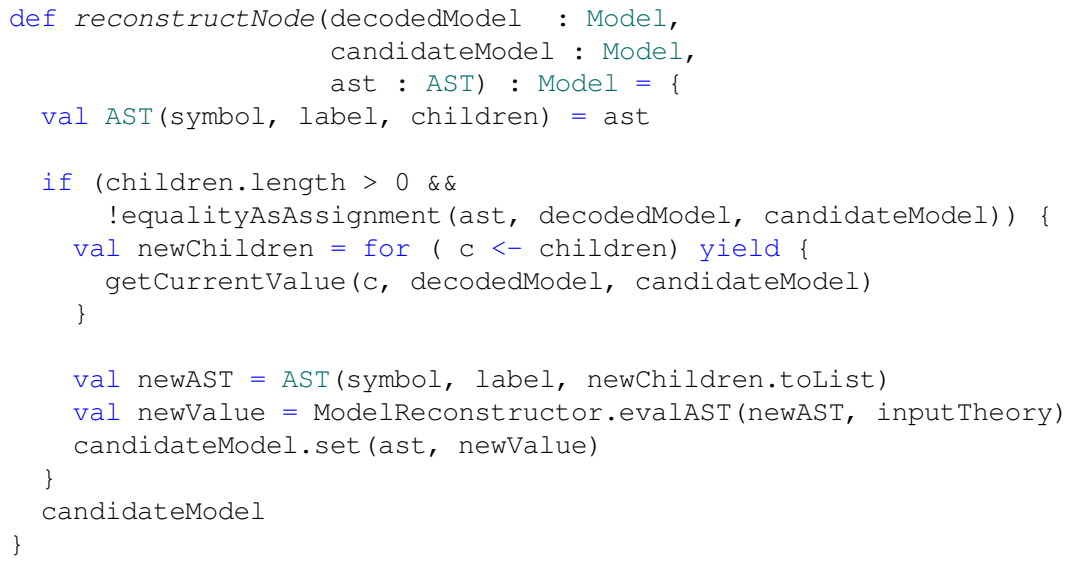

Figure 8. An example of post-order reconstruction, using equality-as-assignment

all predicates of the form $x=y$ or $x=f(\ldots)$, where $x, y$ var variables and $f$ is some operation or predicate. We call these equations definitional. In order to maximise the propagation during the reconstruction, definitional equalities are prioritised over the remaining predicates.

Furthermore, the definitional equalities are sorted based on a topological order of the variables in a graph defined by viewing definitional equalities as directed edges in a graph. An equation of the form $x=f(\ldots)$ generates edges from every variable on the right hands side to $x$, and an equation of the form $x=y$ generates an edge from $x$ to $y$ and one edge from $y$ to $x$. The topological sorting of variables starts with the varibles occuring in definitional equalities, that have the lowest input degree. Their values can be safely copied from the decoded model. The resulting order of variables corresponds to a bottom-up propagation through the formula, that maximises applicatoins of equality-asassignment in the reconstruction. Any cyclic dependencies will be broken, with algorithm picking any variable arbitrarily (we leave it to future work to design a reasonable heuristic). After a topological order of the variables have been established, the equalities ordered according to the variable-ordering.

The model reconstruction performs a bottom-up reconstruction of critical atoms in the topological order of the equalites followed by the remaining predicates. Ordering the predicates in the described manner increases the likelihood of propagation fixing rounding errors introduced by the FPFP encoding.

Model-guided refinement strategy takes place when model reconstruction fails to obtain a model. Model-guided refinement increases the precision of the formula, based on the decoded model and the failed candidate model. The refinement increases the precision of operations, but only so far that a more 


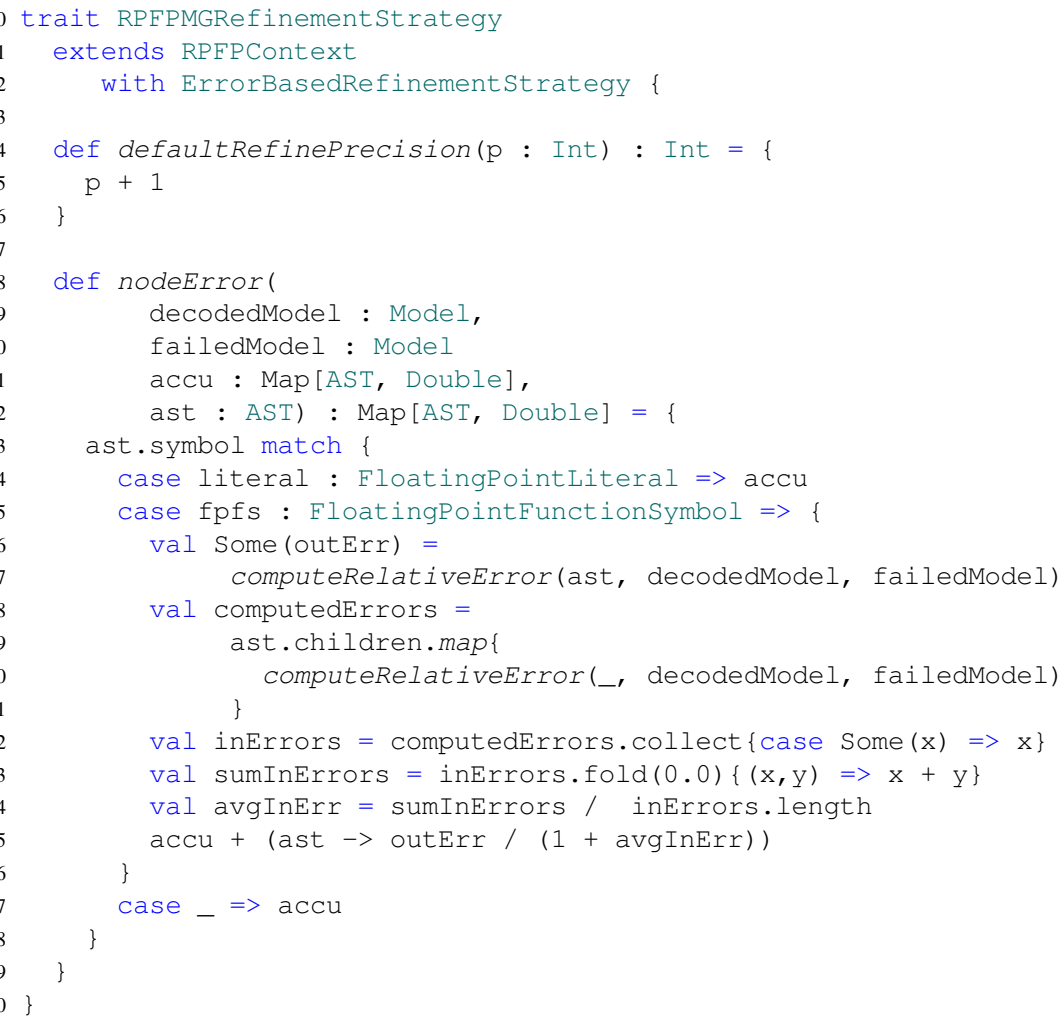

Figure 9. Implementation of a model-guided refinement strategy based on relative errors

precise model is obtained in the next iteration. Comparison of the evaluation of the formula under the two assignments, highlights critical atoms that should be refined. These atoms evaluate to true in the approximate model and to false in the failed candidate model. Since FPA is a numerical domain, it is possible to apply some notion of error to determine which nodes contribute the most to the discrepancies in evaluation and use them to rank the sub-expressions. After ranking, only a portion of them is refined, say $30 \%$. Refinement amounts to increasing precision by some amount, in this case a constant. In general, one could use the error to determine by how much to increase the precision. Since error-based refinement can be applied to any numerical domain, UppSAT implements an error-based refinement strategy, which is instantiated by providing an implementation of the nodeError hook function, shown in Fig. 9. 


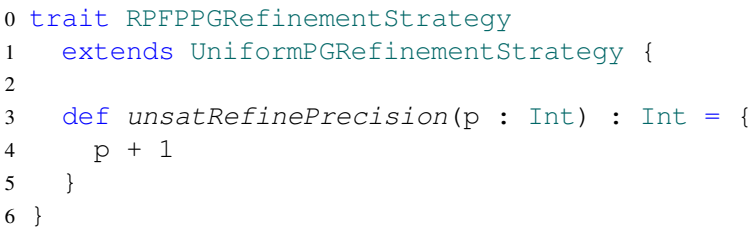

Figure 10. A naive proof-guided refinement strategy uniformly increasing precision

Proof-guided refinement strategy uses proofs of unsatisfiability to refine the formula. Formula can be refined using unsatisfiable cores, when an approximate model is not available. At the moment UppSAT has no support for obtaining cores or proofs from the back-end solvers. Instead, a naïve refinement strategy is used, which increases all the precisions by a constant, shown in Fig. 10.

\section{Approximations in UppSAT}

In this section, we discuss some more general aspects of approximations within the UppSAT framework. In addition to listing alternatives to the components of the RPFP approximation, some implementation details are discussed.

Precision domains are crucial for both the expressiveness of the encoding and the subtlety of the refinement. Precision can be uniform or compositional in terms of their relationship with the formula. Uniform precision assigns a single precision value to the entire formula, whereas compositional precision associates different values with some or all parts of the formula. As we have seen, the RPFP approximation uses a compositional precision, which is associated with variable and function nodes. Uniform precision is used in the BV and RA approximations, which are presented in the next section.

From the perspective of encoding expressivity, precision can be a scalar value or a vector. While in most cases scalar precision suffices, vectors (or tuples) can be used to elegantly encode more expressive approximations. For instance, a pair of precisions associated with an FPA node allow the significand and the exponent to have independent bit-widths. Choosing a suitable precision domain is important, both for the compactness of the definition of approximation in UppSAT and for the performance of the resulting approximating solver. Too crude a precision domain might yield a negligible improvement of performance, while too fine a precision domain might spend too much time wandering through the different approximations.

Encoding and decoding are the heart of the approximation. The two translations are intertwined, a simple elegant encoding is useless if the model cannot 
be translated back in a meaningful way. In fact, the encoding often suggests a natural way of implementing the decoding, since the translations are in a sense inverse. In general, an encoding is just an arbitrary translation of a formula of the input theory to a formula of the output theory; in practice, like for the RPFP approximation, the encoding does not change the overall structure of the formula, but merely adjust the sorts involved. Other approximations might add global constraints in the encoding, e.g., definitional equalities or impose ranges, or they might add or remove nodes in the formula. For instance, the real-arithmetic approximation RA of FPA will not encode the rounding modes, since they do not have an equivalent in real arithmetic. The decoding of a real model needs to produce some reasonable values for the rounding modes somehow. This can, for instance, be done by choosing a pre-selected default value.

To maintain information of the relationship between the original and the encoded formula, UppSAT uses labeled abstract syntax trees. During the encoding, the result of the encoding is assigned the label of the source node in the original formula that it encodes. The labels offer a way to keep track of the translation, since the encoding can be ambiguous to decode. All the approximations presented in this paper are context-independent and node-based, i.e., it is sufficient to specify the translation at the node level. UppSAT offers a pattern for this kind of codec, called Post OrderCodec. Overall, UppSAT can handle a broad range of encodings, that can be specified succinctly within the framework.

Model reconstruction strategies take place entirely in the input theory, and as such can be combined with a number of different encodings (they are independent of the chosen output theory). The reconstruction strategy used by the RPFP approximation is simple in the sense that it only evaluates expressions, and it does not pose satisfiability queries to a solver. A different strategy, along similar lines, might start the reconstruction from the difficult (e.g., non-linear) constraints and then evaluate the remainder of the formula. More complex strategies might use a solver during the reconstruction to search for a model within some $\epsilon$ distance of a decoded (failing) model. A numeric model lifting strategy was proposed by Ramachandran and Wahl [22]. Their method identifies a subset of the model to be tweaked, and instantiates the formula as a univariate satisfiability check. Except for a chosen variable, all the variables in the formula are substituted by their value in the failing decoded model. This approach often quickly patches the candidate model. UppSAT can express these more advanced strategies, but implementation and experiments in this direction have been left for future work.

Refinement strategies use the information obtained either from the models or the proof of unsatisfiability to find a better approximation. In cases when information is scarce (e.g., no proofs are available in case of unsatisfiability), 
or the approximation is very coarse and no useful information can be extracted from a decoded model, a uniform refinement strategy can increase precision of the entire formula. This is the case with fixed-point approximation BV and the proof-guided refinement of the RPFP approximation. In case of numeric domains, a notion of error can be used to determine which terms to refine and by how much [24]. This is the strategy used by the RPFP approximation. In the case of a precision vector for each node in a formula, the error between the decoded and candidate model can be used to refine either the exponent, if the magnitude of the error is large, or the significant if the error is very small.

\section{Other Approximations of FPA}

We have shown in detail the RPFP approximation of FPA, and discussed different components that can be used in general. In this section we outline two further approximations of FPA that have been implemented in UppSAT: the fixed-point approximation BV (Section 1.1.2), encoded as bit-vectors, and the real-arithmetic approximation RA. Both approximations are currently implemented in a more experimental and less refined way than the RPFP approximation, but encouragingly, even simple approximations can give rise to speed-ups compared to their back-end solvers (as shown in Section 7).

\subsection{BV - The Fixed-Point Approximation of FPA}

The idea behind the BV approximation is to avoid the overhead of the rounding semantics and special values of the FPA, by encoding all the FPA values and variables and operations as values and operations of the fixed-point arithmetic.

The BV context. The input theory is the theory of FPA, and the intended output theory is the theory of fixed-point arithmetic. However, since fixedpoint arithmetic is not commonly supported by SMT solvers, we can encode fixed-point constraints in the theory of fixed-width bit-vectors. The precision determines the number of integer and fractional binary digits in the fixed-point representation of a number. For simplicity, at this point we do not mix multiple fixed-point formats in one formula, but instead apply uniform precision in the $\mathrm{BV}$ approximation; as a result, all operations in a constraint are encoded using the same fixed-point sort. As a proof of concept, the precision domain is twodimensional, with the first component $p_{i}$ in a pair $\left(p_{i}, p_{f}\right)$ denoting the number of integral, and the second component $p_{f}$ the number of fractional bits in the encoding, respectively. The precision domain ranges from $(5,5)$ to $(25,25)$, with the maximum element $(25,25)=\top$ being interpreted as sending the original, unapproximated FPA constraint to Z3 as a fall-back solver.

Example 4. Given a variable of precision $(4,5)$, we will have a domain of numbers between 1000.00000 and 0111.11111 , which when interpreted in two's-complement notation are numbers between -8 and 7.96875 . 
The BV codec. A codec describes how values can be converted from the input theory to the output theory, and vice versa. The floating-point operations are in BV encoded as their fixed-point equivalents, which in turn are encoded as bit-vector operations. This process is fairly straightforward, with the exception of the rounding modes and special FPA values. The rounding modes and not-a-number values are omitted by the encoding, while the remaining special values are encoded, with respect to the current precision, either as zero or as the largest or smallest value (in case of infinities). Translation of literal floating-point constants amounts to a representation as the closest value in the chosen fixed-point sort. The decoding consists of converting a fixed-point number to a rational number, followed by conversion to the closest floatingpoint number, with some care taken for the special values.

BV reconstruction and refinement. The $\mathrm{BV}$ approximation uses the same model reconstruction strategy as the RPFP approximation. In contrast, the chosen refinement strategy in the BV approximation is currently very simple: since the precision is uniform, the refinement is also uniform, regardless of whether an approximate model is available or not. At each iteration, the precision is increased by 4 in both dimensions, resulting in addition of 4 bits to both the integral and fractional part of numbers. ${ }^{2}$

\subsection{RA - The Real Arithmetic Approximation of FPA}

The third and possibly most obvious approach to approximate FPA is by encoding into real arithmetic constraints. We present a comparatively simplistic implementation of this kind of approximation, due to the difficulty to refine approximations in real arithmetic in a meaningful way (real arithmetic already represents to infinite-precision arithmetic). Ramachandran and Wahl [22] describe a topological notion of refinement, that requires a back-end solver that handles the combined theory of real arithmetic and FPA. However, solving constraints over this combination of theories is challenging in itself, and efficient SMT solvers are not publicly available, to the best of our knowledge.

RA context. In the RA approximation, the FPA is the input theory, and the output theory is the theory of (non-linear) real arithmetic. The precision domain is a uniform binary domain $\{\perp, T\}$, deciding whether approximation is taking place at all $(\perp)$, or whether the original FPA constraint is sent to a back-end solver (for T; again, the fall-back solver in this case is Z3). Essentially, this is a hit-or-miss approximation, which either will work right away or directly resort to the fall-back solver.

\footnotetext{
${ }^{2}$ This means that the approximation does not really leverage the two-dimensional precision, and that maximal precision of the encoding is reached after at most 5 iterations.
} 
RA codec. The encoding is fairly straightforward, the FPA operations are translated as their real counter-parts, omitting the rounding modes in the process. While the special values can be encoded, currently they are not supported by the RA approximation. FPA numerals are converted to reals, i.e., in the case of normal FPA numbers the resulting real number is $(-1)^{\text {sign }} \cdot$ significand . $2^{\text {exponent }}$. Decoding will translate a real number to the closest FPA numeral.

RA reconstruction coincides with the RPFP reconstruction.

RA refinement is achieved by uniform refinement, and results in the full precision $T$ after a single iteration. In the case of the topological refinement proposed by Ramachandran and Wahl [22], the precision domain would be the same, but the precision itself would be compositional, i.e., a precision would be associated with each node of the formula. Essentially, the precision would represent a switch, deciding whether a node should be encoded in real arithmetic or floating-point arithmetic.

\section{Related Work}

\subsection{Approximations in General}

The concept of abstraction (and approximation) is central to software engineering and program verification, and is increasingly employed in general mathematical reasoning and in decision procedures as well. Frequently only under- and over-approximations are considered, i.e., the formula that is solved either implies or is implied by an approximate formula. Counter-example guided abstraction refinement [7] is a general concept that is applied in many verification tools and decision procedures, even on a relatively low level as in QBF solvers [17], or in model-based quantifier instantiation for SMT [15].

Specific instantiations of abstraction schemes in related areas also include the bit-vector abstractions by Bryant et al. [5] and Brummayer and Biere [4], as well as the (mixed) floating-point abstractions by Brillout et al. [3].

Van Khanh and Ogawa present approximations for solving polynomials over reals [18]. Gao et al. [14] present a $\delta$-complete decision procedure for non-linear reals, considering over-approximations of constraints by means of $\delta$-weakening.

\subsection{Decision Procedures for Floating-Point Arithmetic}

The SMT solvers MathSAT [6], Z3 [21], and Sonolar [19] feature bit-precise conversions from FPA to bit-vector constraints, known as bit-blasting, and represent the currently most commonly used solvers in program verification. As we show in our experiments, bit-blasting can be boosted significantly with the help of our approximation approach. 
A general framework for decision procedures is Abstract CDCL, introduced by D'Silva et al. [11], which was also instantiated for FPA [12, 2]. This approach relies on the definition of suitable abstract domains (as defined for abstract interpretation [8]) for constraint propagation and learning. In our experimental evaluation (Section 7), we compare to two decision procedures for FPA that are implemented in MathSAT; instances of ACDCL and eager translation to bit-vectors. ACDCL can seamlessly be integrated into the UppSAT framework, for instance to solve approximations or to derive an approximation based on abstract domains.

The work presented in this paper builds on previous research on the use of approximations for solving FPA constraints [23, 24]. UppSAT is also close in spirit to the framework presented by Ramachandran and Wahl [22] for efficiently solving FPA constraints based on the notion of 'proxy' theories, which correspond to our 'output theories.' This framework applies a relatively sophisticated method of reconstruction, by applying a fall-back FPA solver to a version of the input constraint in which all but one variables have been substituted by their value in a failing decoded model. Such reconstruction could also be realized in UppSAT, and an implementation in UppSAT and experimental comparison with other reconstruction methods is planned as future work.

A further recent approximation-based solver for FPA is XSat [13]. In XSat, reconstruction of models is implemented with the help of randomized optimization, which results in good performance, but does not give rise to a decision procedure (incorrect sat/unsat results can be produced).

There is a long history of formalization and analysis of FPA concerns using proof assistants, among others in Coq by Melquiond [20] and in HOL Light by Harrison [16]. Coq has also been integrated with a dedicated floating-point prover called Gappa by Boldo et al. [1], which is based on interval reasoning and forward error propagation to determine bounds on arithmetic expressions in programs [10]. The ASTRÉE static analyzer [9] features abstract interpretation-based analyses for FPA overflow and division-by-zero problems in ANSI-C programs.

\section{Experimental evaluation}

In this section we evaluate the effectiveness of the discussed approximations of FPA, when combined with the bit-vectors, real and FPA decision procedures implemented in MathSAT and Z3.

Experimental setup. The evaluation is done on the satisfiable benchmarks of the QF_FP category of the SMT-LIB. Currently, UppSAT does not extract unsatisfiable cores from back-end, and none of the approximations have a meaningful proof-based refinement strategy, so that performance on unsatisfiable problems is guaranteed to be worse than that of the back-end solver. All 


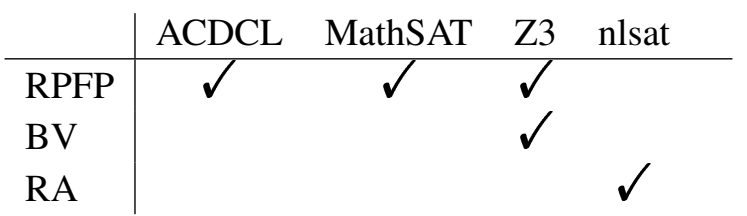

Table 1. Combinations of approximations, shown in rows, and back-end solvers, shown in columns, used to instantiate UppSAT are denoted by $\checkmark$. The instances of UppSAT are named in the format APPROXIMATION(back-end)

experiments were done on an AMD Opteron 2220 SE machine, running 64-bit Linux, with memory limited to $1.0 \mathrm{gb}$, and with a timeout of one hour.

UppSAT instances. Table 1 shows combinations of approximation and backend solver that we evaluate. The UppSAT instances are named in the form of APPROXIMATION(back-end). Note that the back-end needs to implement a decision procedure for the output theory of the approximation. As a consequence, we have three configurations for the RPFP approximation, by using bit-blasting procedures in Z3 and MathSAT and the ACDCL algorithm in MathSAT as decision-procedures for FPA. UppSAT currently lacks support for the bit-vector theory in MathSAT, so for the BV approximation only the bit-vector solver in Z3 is used as the back-end. The back-end for the RA approximation is the nlsat tactic in $\mathrm{Z3}$, since it is the only decision procedure in Z3 and MathSAT to support non-linear constraints over reals.

Investigated questions. In previous work, we have observed that the RPFP approximation improves performance of bit-blasting implemented in the Z3 SMT solver [24]. Here we seek to reproduce those results, but also to see whether similar behavior can be observed with other implementations and algorithms. We were interested in answering the following research questions:

- Is the positive effect of the RPFP approximation on performance of the bit-blasting approach for FPA independent of the implementation?

- Does the RPFP approximation have a positive effect on the ACDCL algorithm for FPA?

- What is the impact of approximations on the state-of-the-art for the theory of FPA?

To answer these questions, we compare the performance of the back-ends and the UppSAT instances on 130 non-trivial $^{3}$ satisfiable benchmarks of the QF_EP category of the SMT-LIB benchmarks. On each benchmark, solvers were assigned a rank based on their solving time, i.e., if a solver had the small-

\footnotetext{
${ }^{3}$ The regression tests in the wintersteiger family were ignored for the evaluation.
} 


\begin{tabular}{|c|c|c|c|c|c|c|c|c|}
\hline & acdcl & mathsat & $\mathrm{z} 3$ & $\begin{array}{l}\text { BV } \\
\text { (z3) }\end{array}$ & $\begin{array}{l}\text { RPFP } \\
\text { (acdcl) }\end{array}$ & $\begin{array}{c}\text { RPFP } \\
\text { (mathsat) }\end{array}$ & $\begin{array}{c}\text { RPFP } \\
(\mathrm{z} 3)\end{array}$ & $\begin{array}{c}\text { RA } \\
\text { (nlsat) }\end{array}$ \\
\hline Solved & 86 & 99 & 97 & 91 & 78 & 101 & 101 & 90 \\
\hline Timeouts & 44 & 31 & 33 & 39 & 52 & 29 & 29 & 40 \\
\hline Best & 65 & 4 & 6 & 9 & 3 & 9 & 9 & 4 \\
\hline Average Iterations & - & - & - & 2.69 & 3.59 & 3.16 & 3.02 & 1.85 \\
\hline Max Precision & - & - & - & 23 & 2 & 1 & 2 & 110 \\
\hline Average Rank & 3.81 & 5.40 & 6.33 & 5.42 & 5.32 & 4.38 & 4.53 & 6.60 \\
\hline Total Time (s) & 10071 & 16748 & 34526 & 11979 & 8448 & 8279 & 14992 & 27169 \\
\hline Average Time (s) & 117.10 & 169.17 & 355.94 & 131.64 & 108.30 & 81.97 & 148.43 & 301.87 \\
\hline Only solver & 1 & 0 & 2 & 0 & 0 & 1 & 0 & 0 \\
\hline
\end{tabular}

Table 2. Comparison of solver performance in terms of number of benchmarks solved and relative ranking of runtimes. The first three columns give numbers for the backends alone, while the other columns consider the configurations specified in Table 1.

est solving time, it was assigned rank 1, the solver with the next smallest solving time rank 2 , etc.

The results are summarized in Table 2, and a more detailed view of runtimes is provided by the cactus plot shown in Figure 11. Table 2 shows, for each solver, the number of benchmarks solved within the 1 hour timeout, the number of timeouts, the number of instances for which the solver was fastest, the average number of refinement iterations on solved problems, the number of benchmarks for which refinement reached maximum precision $T$, the average rank, the total time needed to process all benchmarks (excluding timeouts), the average solving time (excluding timeouts), and the number of unique instances only solved by the respective solver.

Discussion. We can observe that the RPFP approximation combined with bit-blasting, either in Z3 or MathSAT, solves the largest number of instances. When comparing the average rank, MathSAT comes out as the marginally better choice of back-end. This is expected, based on the performance on the back-ends themselves. All the configurations shine on at least a few benchmarks, indicating that the approximations do offer an improvement. Furthermore, the ACDCL algorithm outperforms all the other solvers on 65 benchmarks, which is also indicated by the lowest average rank, but it solves fewer benchmarks that the bit-blasting approaches in total.

Looking only at the approximations, we can see that on average the benchmarks are solved using around three iterations. The notable exception is the RA approximation, which performs at most two iterations, the RA approximation and the full FPA semantics. This indicates that for many of the benchmarks, full-precision encoding is not really necessary, since the RPFP approximation rarely reaches maximum precision. However, the BV and RA approximations reach maximal precision more often. In their defense, both BV and RA approximations are presented as a proof of concept, since neither has tailored reconstruction and refinement strategies. 
Virtual portfolios. To compare the impact of the approximations on the state-of-the-art, we compare a virtual portfolio of the back-end solvers alone, and a virtual portfolio of both the back-ends and the UppSAT instances. Table 3 shows the number of benchmarks solved, the number of timeouts, and the total and average solving time. The addition of the UppSAT instances allows only two more benchmarks to be solved, compared to the back-end portfolio. However, the total solving time is improved dramatically.

$\begin{array}{lcc} & \text { Virtual Portfolio (Back-end) } & \text { Virtual Portfolio (All) } \\ \text { Solved } & 110 & 112 \\ \text { Timeouts } & 20 & 18 \\ \text { Total time } & 25135 & 12516 \\ \text { Average time } & 228.50 & 111.75\end{array}$

Table 3. Comparison of virtual portfolio solver performance.

Cactus plot. To complement the aggregated data, the cactus plot in Figure 11 shows on the $X$ axis how many instances can be solved in the amount of time shown on the Y axis, by each of the solvers and the portfolios. The UppSAT instances are shown using full lines, while the back-ends are presented using dashed lines. The colors denote the same back-end, e.g., mathsat and RPFP(mathsat) are both colored green.

It corroborates that the ACDCL algorithm is very efficient in solving many benchmarks, solving as many as 68 in less than 10s, however, eventually it gets overtaken by the other solvers. Looking more closely at the RPFP approximation, we can conclude that it improves performance of bit-blasting considerably, regardless of the implementation (MathSAT or Z3). On the other hand, RPFP seems to hinder, rather than help, the already very efficient ACDCL algorithm. ${ }^{4}$ Furthermore, the virtual portfolios are also shown. While both portfolios solve more instances than any individual solver, the portfolio based on the back-end solvers and the UppSAT instances is a clear winner, showing the impact of presented approximations on the state-of-the-art.

Detailed comparisons. To complete the picture, Figure 12 shows scatter plots of each approximation against the corresponding back-end. The $\mathrm{X}$ axis denotes the solving time of the UppSAT instance, while the $\mathrm{Y}$ axis denotes the solving time of the back-end. Maximum value along either axes denotes a timeout. Benchmarks on the diagonal show same solving time, above the diagonal the UppSAT instance is faster, and below the diagonal the back-end

\footnotetext{
${ }^{4}$ Earlier experiments using the stable version 5.4.1 of MathSAT have shown similar effects of the RPFP approximation to those on the bit-blasting methods. However, overall the performance results were not consistent with performance of MathSAT in previous publications, and indicated a bug. We thank Alberto Griggio for promptly providing us with a corrected version of MathSAT, which we use in the evaluation.
} 


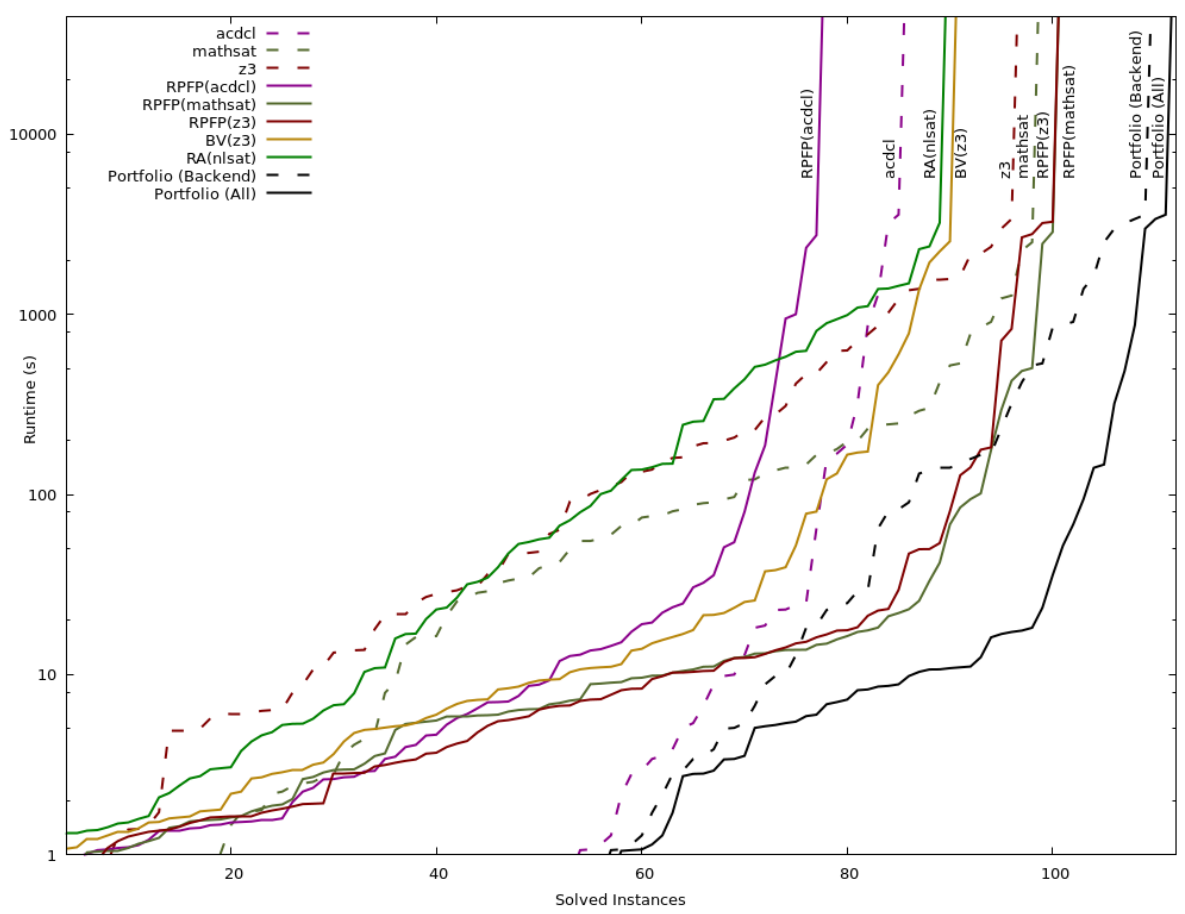

Figure 11. Cactus plot shows how many instances can be solved by a solver in a given amount of time. Comparison of all solvers on the satisfiable SMT-LIB benchmarks.

is faster. In the case of $\mathrm{BV}$ and RA approximations, we compare against $\mathrm{Z3}$ as the baseline, since their respective back-ends do not support the theory of FPA.

The plots featuring Z3 and MathSAT as the back-end seem to show superlinear speedup as a result of approximation, which is suggested by the trend among the instances not being parallel to the diagonal. The cause of this phenomenon is not entirely clear at this point, and will need further investigation; one hypothesis is that approximation is more effective on larger than on smaller FPA problems. Compared to the experiments in [24], the improved results are likely due to strengthening of the model reconstruction strategy.

As evidenced by the previous data, the ACDCL algorithm does not benefit from the RPFP approximation, in terms of performance. However, the approximation does solve some instances without reach of the plain ACDCL procedure.

In the cactus plot, the BV approximation shows improvement in performance over the bit-blasting back-ends, but eventually falls behind in the number of solved instances. Compared to the Z3 back-end, we can see traces of super-linear speed up, but there are also cases where the BV approximation times out. Considering that the BV approximation consists almost entirely of 

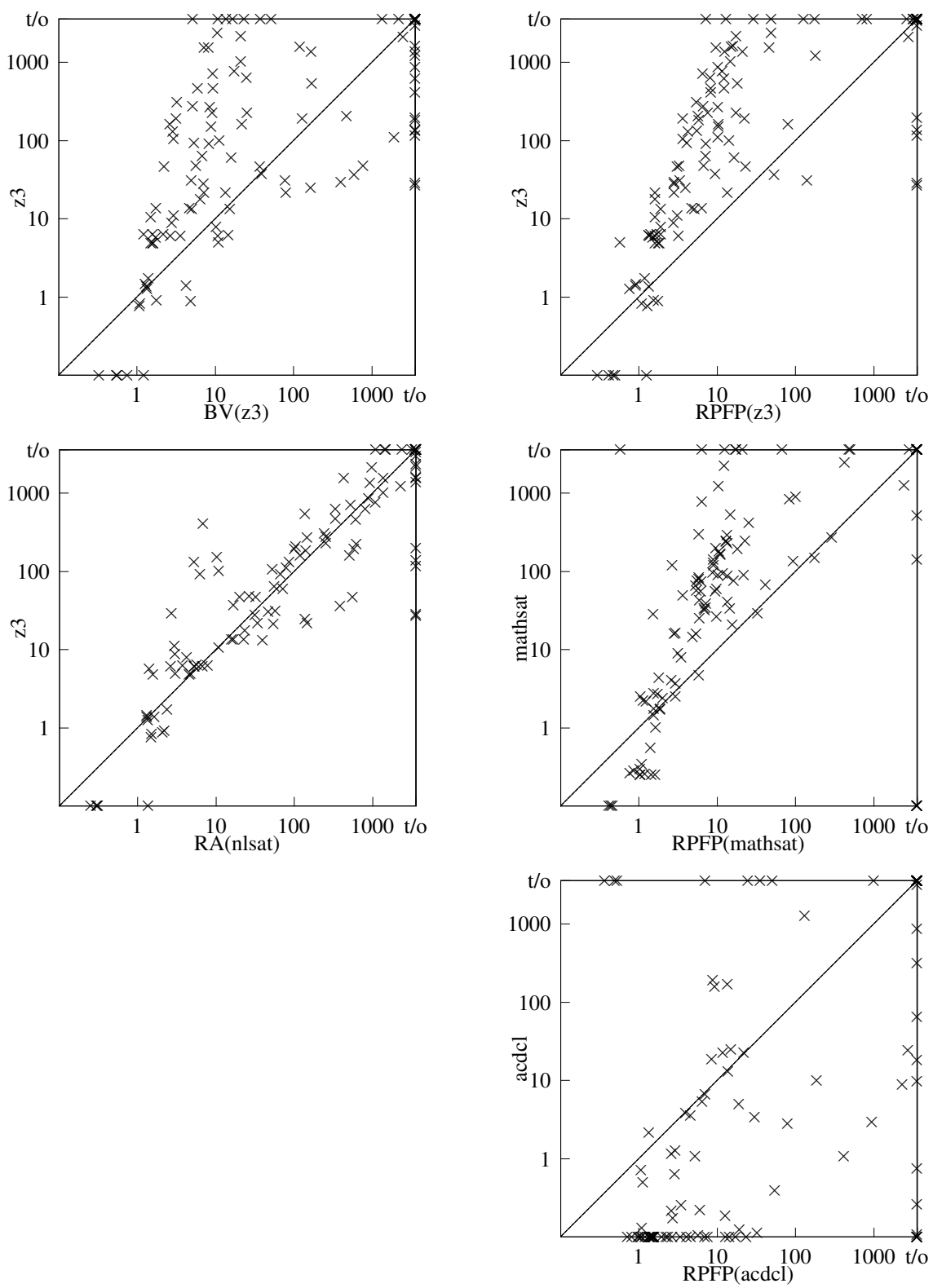

Figure 12. Comparison of runtime performance of UppSAT instances against the corresponding back-end solver. 
the encoding, and that it lacks tailored reconstruction and refinement strategies, we find these results to be quite promising.

The RA approximation also shows some improvement in performance compared to the bit-blasting method. However, we can see from Table 2 that it reaches maximum precision on all but 20 benchmarks, indicating its lack of maturity. It is interesting, however, that it does outperform all the other solvers on a few benchmarks. One approach would be to allow mixed constraints of FPA and RA, as proposed by Ramachandran et al. [22], however none of the off-the-shelf SMT solvers support this theory combination in a meaningful way yet.

Finally, a comparison of the three back-end solvers, and of the corresponding combination of back-end solver and RPFP approximation, is given in Fig. 13 in the Appendix.

\section{Conclusion and Future Work}

We have presented a methodology and new framework, UppSAT, for implementing approximating SMT solvers. The experimental evaluation demonstrates, what has been showed earlier, the efficiency of approximations as well as provides a simple implementaiton. The presented framework does not only yield a significand preformance improvement in comparison with the backends, it does so with a modular code allowing for easy combinations of different strategies.

Approximation configurations presented here (RPFP(z3), RPFP(mathsat)) are shown to be state-of-the art in handling formulas in FPA, where they improve their performance of the respective back-end. For ACDCL this is not the case, however, indicating that perhaps a different method of approximation should be utilized. A strenght of the UppSAT framework is that if a new SMT-solver would be improved on FPA formulas, it can easily be integrated into the approximation framework, perhaps improving the underlying backend even further.

The clear direction for improving UppSAT is to extend the general framework with more abstract strategies, e.g., retrieve multiple models from an approximate formula and/or apply multiple different reconstruction strategies on approximate models. Currently, majority of the time is spent on looking for models which means there is plenty of room to make more sophisticated strategies in the framework.

Another big challenge is to extend UppSAT to be able to handle unsatisfiable formulas efficiently. Currently, the proof refinement is naive uniform refinement, but there is a potential to do much more intelligent refinement. This research has been focused on finding models, but it is very interesting to investigate how unsatisfiability proofs from approximated formulas can be utilized for refinement. 
The fixed point and real arithmetic approaches are presented here as a proof of concept. They are simple and not much effort went into instantiating the framework for these approximations. However, the results shows that even uncomplicated approaches can be competitive; this opens up the line of future work to design tailored refinement and reconstruction strategies.

We present virtual portfolio solvers, which show some kind of optimal choice among which solver to chose. It would be interesting to create a real portfolio solver and see how well it can be tuned to pick the correct solver (e.g., by machine learning algorithms) as well as utilize parallelism.

\section{References}

[1] Boldo, S., Filliâtre, J.C., Melquiond, G.: Combining Coq and Gappa for certifying floating-point programs. In: Intelligent Computer Mathematics (Calculemus); MKM/CICM. LNCS, vol. 5625. Springer (2009)

[2] Brain, M., D’Silva, V., Griggio, A., Haller, L., Kroening, D.: Deciding floating-point logic with abstract conflict driven clause learning. FMSD (2013)

[3] Brillout, A., Kroening, D., Wahl, T.: Mixed abstractions for floatingpoint arithmetic. In: FMCAD. IEEE (2009)

[4] Brummayer, R., Biere, A.: Effective bit-width and under-approximation. In: EUROCAST. LNCS, vol. 5717. Springer (2009)

[5] Bryant, R.E., Kroening, D., Ouaknine, J., Seshia, S.A., Strichman, O., Brady, B.A.: Deciding bit-vector arithmetic with abstraction. In: TACAS. LNCS, vol. 4424. Springer (2007)

[6] Cimatti, A., Griggio, A., Schaafsma, B.J., Sebastiani, R.: The MathSAT5 SMT solver. In: TACAS. LNCS, vol. 7795. Springer (2013)

[7] Clarke, E.M., Grumberg, O., Jha, S., Lu, Y., Veith, H.: Counterexampleguided abstraction refinement. In: CAV. LNCS, vol. 1855. Springer (2000)

[8] Cousot, P., Cousot, R.: Abstract interpretation: A unified lattice model for static analysis of programs by construction or approximation of fixpoints. In: Proceedings, Fourth ACM Symposium on Principles of Programming Languages, Los Angeles, USA. pp. 238-252. ACM Press (1977)

[9] Cousot, P., Cousot, R., Feret, J., Mauborgne, L., Miné, A., Monniaux, D., Rival, X.: The ASTREÉ analyzer. In: ESOP. LNCS, vol. 3444. Springer (2005)

[10] Daumas, M., Melquiond, G.: Certification of bounds on expressions involving rounded operators. ACM Trans. Math. Softw. 37(1) (2010)

[11] D'Silva, V., Haller, L., Kroening, D.: Abstract conflict driven learning. In: POPL. ACM (2013)

[12] D’Silva, V., Haller, L., Kroening, D., Tautschnig, M.: Numeric bounds 
analysis with conflict-driven learning. In: TACAS. LNCS, vol. 7214. Springer (2012)

[13] Fu, Z., Su, Z.: XSat: A fast floating-point satisfiability solver. In: Chaudhuri, S., Farzan, A. (eds.) Computer Aided Verification - 28th International Conference, CAV 2016, Toronto, ON, Canada, July 17-23, 2016, Proceedings, Part II. Lecture Notes in Computer Science, vol. 9780, pp. 187-209. Springer (2016), https : / doi .org/10.1007/ 978-3-319-41540-6

[14] Gao, S., Kong, S., Clarke, E.M.: dReal: An SMT solver for nonlinear theories over the reals. In: CADE. LNCS, vol. 7898. Springer (2013)

[15] Ge, Y., de Moura, L.: Complete instantiation for quantified formulas in satisfiability modulo theories. In: CAV. LNCS, vol. 5643. Springer (2009)

[16] Harrison, J.: Floating point verification in HOL Light: the exponential function. TR 428, University of Cambridge Computer Laboratory (1997), available on the Web as http://www.cl.cam.ac.uk/ jrh13/papers/tang.html

[17] Janota, M., Klieber, W., Marques-Silva, J., Clarke, E.: Solving QBF with counterexample guided refinement. In: SAT. LNCS, vol. 7317. Springer (2012)

[18] Khanh, T.V., Ogawa, M.: SMT for polynomial constraints on real numbers. In: TAPAS. Electronic Notes in Theoretical Computer Science, vol. 289 (2012)

[19] Lapschies, F., Peleska, J., Gorbachuk, E., Mangels, T.: SONOLAR SMTsolver. In: Satisfiability modulo theories competition; system description (2012)

[20] Melquiond, G.: Floating-point arithmetic in the Coq system. In: Conf. on Real Numbers and Computers. Information \& Computation, vol. 216. Elsevier (2012)

[21] de Moura, L., Bjørner, N.: Z3: An efficient SMT solver. In: TACAS. LNCS, vol. 4963. Springer (2008)

[22] Ramachandran, J., Wahl, T.: Integrating proxy theories and numeric model lifting for floating-point arithmetic. In: 2016 Formal Methods in Computer-Aided Design, FMCAD 2016, Mountain View, CA, USA, October 3-6, 2016. IEEE (2016)

[23] Zeljic, A., Wintersteiger, C.M., Rümmer, P.: Approximations for model construction. In: Demri, S., Kapur, D., Weidenbach, C. (eds.) Automated Reasoning - 7th International Joint Conference, IJCAR 2014, Held as Part of the Vienna Summer of Logic, VSL 2014, Vienna, Austria, July 19-22, 2014. Proceedings. Lecture Notes in Computer Science, vol. 8562, pp. 344-359. Springer (2014), https : / / doi .org/10.1007/ 978-3-319-08587-6

[24] Zeljic, A., Wintersteiger, C.M., Rümmer, P.: An approximation framework for solvers and decision procedures. J. Autom. Rea- 
soning 58(1), 127-147 (2017), https://doi.org/10.1007/ s10817-016-9393-1 

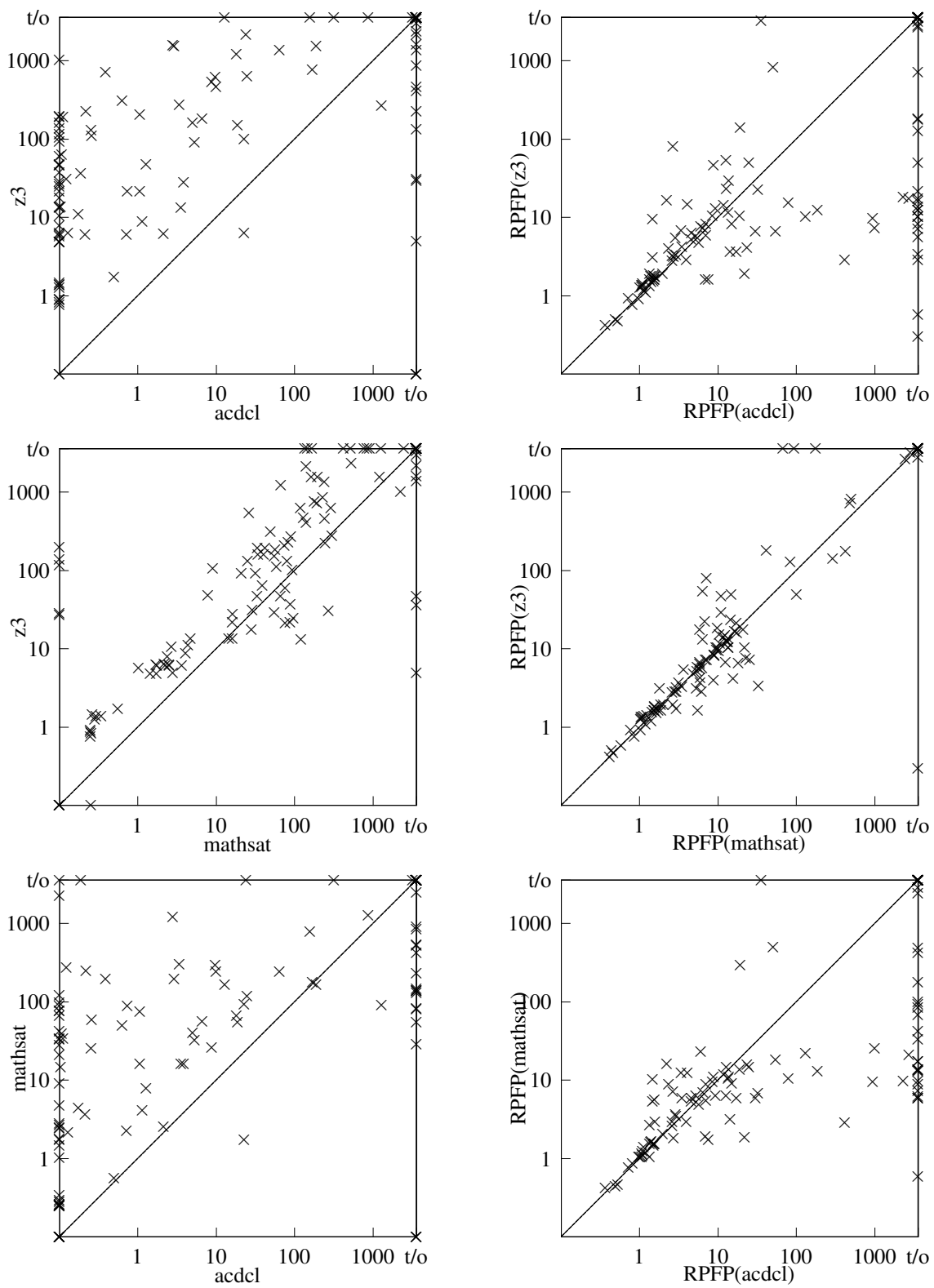

Figure 13. Comparison of runtime performance the back-end solvers, without approaximation (left) and using RPFP approximation (right). 